\title{
Notas acerca de la Urea
}

\author{
POR el Dr. MANUEL A. VELASQUEZ
}

Catedratico de Qulmica Médics en la Facultad de Medictna.

A urea, $\mathrm{C}=\mathrm{O}<_{\mathrm{HN}}^{\mathrm{NH}}$ : Peso molecular $=60$, es considerada en justicia como el producto principal del metabolismo de las proteinas y como el más interesante de los constituyentes nitrogenados de la orina y como el más importante también, pues contiene un $45 \%$ de azoe y toma para ella el 85 a $90 \%$ del nitrogeno total de la orina. Contribuye a afirmar este concepto de la importancia de la úrea, el hecho de que casi el $90 \%$ del nitrogeno introducido en la economía con los alimentos es eliminado por la orina bajo la forma de urea.

La urea se forma en el hígado, por la unión del COa con grupos aminados (leucina). El higado constituye la sede principalisima de la síntesis de la urea; pues el riñón parece no tomar participación alguna en el metabolismo del nitrogeno y limita su funcion a excretar los productos azoados de desasimilación que le acarrea la sangre. A despecho de esta creencia, tan generalizada como poco discutida, en diciembre del año de 1915 Chevalier y Chabanier han insinuado el hecho, muy sugestivo, muy interesante, de la existencla de una gran cantidad de urea en la sustancia cortical del riñon, ocupando las células de los tubuli contorti. (1)

(1) Paul Gmivalier et H. Ghabakier. - Sur la localization de lo uroe dans le rein.-Comptes Rendus de la Socite de Btologie.-pag. 689. Paris 1915. 
Admítese la posibilidad de que el bazo, los ganglios linfáticos y otras glándulas secretantes contribuyan, en escala más o menos modesta, a la formación de urea en el organismo.

La cantidad de urea elimirada por la orina en 24 horas está comprendida entre el 1 y el 3 por ciento: es de 20 a 30 gramos en el adulto, durante las 24 horas: es de 0.40 por kilo de peso (cifra algo menos en los ancianos y en los sujetos de abundante panículo adiposo). Cuando la cantidad de cloruros, de la orina es normal, la urea constituye la mitad de los solidos totales de la orina (coeficiente $=50: 100$ ).

El nitrógeno total eiiminado se desdobla en $\mathrm{N}$ de la orina $(95 \%)$ y $\mathrm{N}$ de las heces $(5 . \%)$

Del nitrógeno urinario contiene la urea un 85 a $95 \%$ del nitrogeno total, correspondiente el $10 \circ 15 \%$ restante al amoniaco y a otros derivados: ácido úrico ( 1 a $3 \%$ ) y el nitrogeno extractivo (1) $(10$ a $15 \%)$.

Los niños eliminan 1 gramo de urea por kilo de peso (3 a 5 años): 0.8 gramos (8 a 11 años); 0.4 a 0,6 (13 a 16 años). El recién na cido elimina 0.23 gramos por cada kilo de su peso.

Fisiologia.-La eliminación de la urea aumenta: con la alimentación, singularmente cuando los alimentos son ricos en nitrógeno; después de ejercicios mentales ofísicos; después de los baños calientes;aconsecuencia de la estimulación eléctrica. La mayor proporción de iurea se elimina seis horas después de las comidas y la proporción minima durante las primeras horas de la mañana.

La eliminación de urea disminuye: en todos los casos en que disminuye la asimilación de proteinas: en los ayunadores, en los sujetos que se alimentan insuficientemente, en aquellos que extreman su devoción por la dieta vegetariana, en aquellos que viven una vida sedentaria, en los que siguen una dieta lactea prolongada, en los que ingieren, en exceso y continuadamente, líquidos. Tambien disminuye la urea durante la menstruacion y durante el embarazo. Disminuye, así mismo, merced a la acción de medicamentos diuréticos y de otros que alteran en alguna manera la funcionalidad hepática.

Patologia.-La eliminacion de urea aumenta: en todos aquellos estados patológicos que activan o contribuyen a activar el metanina.

(1) Comprende toilos los demás compuestos nitrogenados, estimados en creati- 
bolismo orgánico; por consiguiente, es de observarse una mayor eliminación de urea en los procesos febriles, singularmente en la malaria, en la neumonia. Hállasela igualmente aumentada en las manifestaciones convulsivas de las grandes neurosis: histeria y epilepsia, durante el curso de la uremia, en la leucocitemia, en el escorbuto, en los casos en los que se opera una reabsorción de exudados y trasudados, como sucede en las nefritis acompañadas de reabsorción de los derrames, en la gota, en ciertos trastornos gistro intestinales, etc.

La disminución de urea en la orina se debe a un desorden funcional de índole hepática, a una retención de la urea en la sangre por impermeabilidad renal, excepción hecha de los casos en que la disminución es resultado exclusivo de una deficiente ingestión de.proteinas. Es por esta razón que la disminución apreciable de la relación normal existente entre el nitrógeno ureico y el nitrógeno total, debe ser mirada como un signo evidente de insuficiencia hepática (ureogenética).

La disminución de la cantidad de urea eliminada por la orina tiene lugar en todas aquellas circunstancias en las cuales ocurre una perturbacion, más o menos profunda, de los procesos de nutricion: ocurre dicha disminución en los procesos degenerativos del hígado (atrofia amarilla, cirrosis, carcinoma, icteria aguda febril); dicha disminucion suele suceder a las crisis de las enfermedades febriles agudas, al inicio de alguna de las cuales, como la fiebre tifoidea, suele presentarse también. Preséntase, así mismo, en el curso de muchas enfermedades crónicas como las nerviosas y aquellas en las cuales el síntoma dolor figura en primera línea. Preséntase también en los casos de tumores uterinos y ovarianos, suele acompañar a la for mación de exudados y trasudados, a las nefritis aguda y subaguda, a la nefritis intersticial; al colera, a la fiebre amarilla y a algunas enfermedades en las cuales se presentan vómitos abundantes, diarreas y sudores profusos

Notas clinicas.-En los estados febriles agudos la cifra de urea eliminada puede adquirir límites muy altos, llegando a 85 gramos en las 24 horas: En la neumonia la cifra media de urea eliminada suele ser de 30 gramos, llegando, en casos excepcionales, hasta 48 . Es mayor aun la cifra de eliminación de la urea en la diabetis mellitus, en la cual alcanza con relativa frecuencia la cifra de 50 gramos, llegando, a título excepcional, a 130 gramos en las 24 horas. También se observa un aumento notable en la cantidad de urea eliminada en 
la fiebre tifoidea, en la cual no es raro ver subir dicha cifra a la de 60 gramos en las 24 horas.

Una disminución de la urea eliminada en el periodo inicial de una neumonia permite pronosticar, con muchas probabilidades de acierto, una convalescencia prolongada, de duración suficientemente larga para que el organismo pueda rehacerse del serio quebranto sufrido.

En algunas enfermedades de sede ovariana, singularmente en el cancer de dichos organos, la cantidad de urea eliminada puede bajar a 15 o 18 gramos en las 24 horas. Es aun menor la cantidad de urea eliminada en la anemia, en la cual puede llegar a 10 gramos en las 24 horas: a este mismo bajo nivel puede llegar durante el curso de ciertos embarazos. En las enfermedades renales crónicas y avanzadas, una disminución de urea bien manifiesta es signo de mal presagio, pues, suele ser el anuncio del mayor peligro que debe temer el práctico que asista a uno de dichos enfermos: el peligro de la uremia. En el curso de las enfermedades hepáticas, la disminucio de la urea es mucho más marcada que la del nitrógeno total, observase al mismo tiempo, un aumento en la eliminación del amoniaco. es por esta circunstancia que se recomienda al práctico la impor-" tancia de conocer exactamente la proporcion existente entre la urea y el amoniaco eliminados.

La disminución de urea, unida a la presencia en la orina de leucina y tirosina, es todo un indicio en favor de la sospecha de una atrofia a marilla del hígado.

De la misma manera, una disminución de la urea, unida a disminución del nitrógeno total, a disminución de la relación normal entre el nitrógeno ureico y el nitrógeno total, a disminución de los cloruros y de los fosfatos y a reacción neutra o alcalina de la orina, constituye carácter de cierta importancia en el diagnóstico del cáncer, sea cual fuera el organo que hubiere elegido para implantarse y desarrollarse.

Si bien la disminución de la úrea eliminada puede llegar hasta su ausencia total en el período de fatal terminación de las enferme. dades febriles agudas, no debe concederse valor tan amplio a la exclusiva disminución de la cantidad de úrea durante 24 horas del curso de una enfermedad cualquiera: dicha disminución por si sola no es un signo infalible de intoxicación urémica.

Dosaje de la úrea.-Este dosaje, muy interesante en Clinica, puede llevarse a cabo según diversos procedimientos:

a) Por la densidad.-Existiendo una relacion constante entre la densidad de la orina normal (sin albúmina, sin glucosa, otc.) 
y la cantidad de úrea eliminada,es posible determinar esta última por medio de la medida de la densidad de la orina en exámen.

En ausencia de glucosa, en ausencia de albúmina o en present cia de esta no superior a la cifra de $0.20 \%$, con una cifra de cloru-! ros correspondiente a la normal, puede hacerse el cálculo con'sujecion a las cifras siguientes:

Densidad $=1014=1 \%$ de úrea

Densidad $=1020$ a $24=2 \%$ a $2.5 \%$ de úrea

Densidad $=1028$ a $30=3 \%$ de úrea

b) Por descomposición gasométrica.-Se trata de la acción, en frío, de los hipocloritos o hipobromitos alcalinos sobre la úrea, poniendo en libertad el $\mathrm{N}$, conforme a la siguiente reaccion:

$$
\begin{aligned}
& \mathrm{CO}<\mathrm{NH}^{2}+3 \mathrm{Na} \mathrm{BrO}=3 \mathrm{NaBr}+\mathrm{CO}+2 \mathrm{H}^{2} \mathrm{O}+2 \mathrm{~N} \\
& \begin{array}{c}
\text { (urea) de soda) } \\
\text { de sodio) }
\end{array}
\end{aligned}
$$

Con el carbonato de amoniaco, se verifica la misma reaccion:

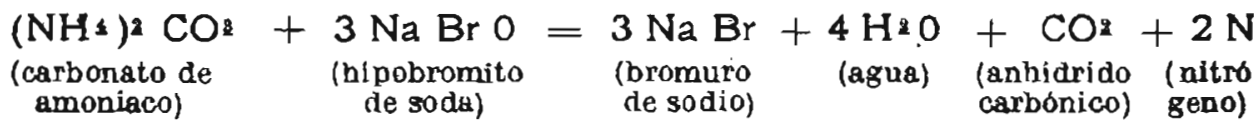

El nitrogeno desprendido medido en centímetros cúbicos da la cantidad de úrea en uno y otro caso:

1 c.c. de $\mathrm{N}=0,0027$ gramos de úrea. 1 gr. de Urea $=374$ c.c. de $\mathrm{N}$.

Un centímetro cúbico de nitrógeno, a $20^{\circ} \mathrm{C}$ y a $750 \mathrm{~mm}$. de presibn corresponde en gramos de úrea por litro a 0.963 .

Para la preparación del reactivo, suelen emplearse varias formulas de las que insertamos las siguientes, advirtiendo que concedemos nuestras preferencias a la segunda en razón de su mejor y más prolongada conservacion:

$$
\begin{aligned}
& \text { No } 1 \text { - Agua de Javel } \\
& \text { Bromuro de potasio } \\
& \mathrm{No} 2 a)-\mathrm{NaOH} \\
& \mathrm{H} \approx \mathrm{O} \\
& \text { b) }-\mathrm{Br} \\
& 20 \text { c. c. } \\
& 1 \mathrm{gr} \text {. } \\
& \text { (Job. y Clarens) } \\
& 100 \mathrm{gr} \text {. } \\
& \mathrm{KBr} \text { una parte en peso de cada uno. } \\
& \mathrm{H}^{2} \mathrm{O} \text { ocho partes. }
\end{aligned}
$$

Mezclese partes iguales de $a$ y de $b$ ( 5 c.c.) y agréguese agua 10 c.c. De esta manera se obtiene una solución de hipobromito de sodio para el dosaje de la úrea en el ureometro.-(RICE.) 
N.03- Solución de hipobromito:
Agua destilada
50 c. c.
Legía de soda 20
Bromo
$\% 50$ c. c. (1.)
2 c.c.

Si se adopta para el dosaje gasométrico el ureometro de MoREIGNe se toman 2 centímetros cúbicos de orina por 10 de hipobromito; si se adopta otro de los ureometros que señalaremos después, se toma solamente un centímetro cúbico de la orina en análisis.

c)-Método de Fowler (2)-Este procedimiento está fundado en el hecho de que cada grado de densidad-que pierde la orina al eliminarse el nitrogeno de la úrea, por acción del hipoclorito, corresponde a la cifra de 0'77\% de úrea. Se emplea el Licor de Labarráque:

Clorinated Lime

Agua destilada
500 gramos

5 litros. (Peso específico 1.055)

Cada gramo de esta mezcla mezclado con 0'5 de yoduro de potasio, disuelto en el agua, produce, cuando se acidula con un centímetro cúbico de ácido clorhídrico, una coloración rojo bruna que requiere para descargar su color no menos de 5 a 6 centímetros cúbicos de solución $\frac{n}{10}$ de tiosulfato de soda, que corresponde a $2 \%$ de cloro estimable. La solución debe dar, cuando esté recientemente preparada, más o menos $3 \%$ de cloro.

Procedimiento.-La tócnica es la siguiente: Tómase un volú: men de orina, cuya densidad se anota; agrégasele siete volúmenes del Labarraque cuya densidad se anota también. Espérase la descomposición de la urea y cuando esta descomposicion ha tenido - lugar (unas dos horas después de realizada la mezcla) se toma la i densidad de esta mezcla.

Se multiplica la densidad del licor de Labarraque por la cifra 7; se agrega a este producto la densidad de la orina; se divide el resultado así obtenido por la cifra 8 ; a este cuociente se le resta la densidad de la mezcla después de la decomposición de la urea. Finalmente, esta diferencia se multiplica por la cifra 0'77 y el resultado de esta multiplicación dá el tanto por ciento de urea. Un ejemplo aclarará estas operaciones, aparentemente complicadas $y$, en realidad, de una gran sencillez:

Supongamos haber anotado las siguientes cifras:

(1) Legia de soda a $36^{\circ}$ Beaume $=40$ grms Na OH por 100 c.c. de agua. p. 29.

(2) PURDY.-Practical Uranalysis and Urinary Diagnosis.-Philadelphia 1909 
Densidad del Licor de Labarraque..... '1027

Densidad de la orina............... 1020

Densidad después de la descomposición.. 1022

Las operaciones por efectuar, serán las siguientes:

$$
\begin{array}{rrrlr}
27 \times & 7 & = & 189 \\
189 & + & 20 & = & 209 \\
209 & 8 & = & 26 \\
26 & \times & 22 & = & 4 \\
4 \times & 0.77 & = & 3.08
\end{array}
$$

La cifra 3.08, obtenida con gran facilidad, reprešentará el tanto por ciento de la urea en la orina examinada.

Dosaje aproximado del nitrógeno de la orina:-Muchas veces le será demandado al químico el valor aproximado del nitrogeno en la orina. En tales casos, procédase en la siguiente forma: (1)

Se toma 10 centímetros cúbicos de orina en una copa y se le agrega, por medio de una bureta graduada en décimos de centímetro cúbico, una solución titulada de nitrato de mercurio (71.48 de Mercurio). Usase como reactivo indicador unas gotas de soda colocadas en un plato de fondo oscuro. La reacción final es la formación de un precipitado amarillo permanente. Se anota el número de centímetros cúbicos de solución gastados y se multiplica este número por la cifra 0.04 y se obtiene la cantidad de nitrogeno. Así, por ejemplo, si han sido necesarios 13 centímetros cúbicos de la solución de nitrato de mercurio, la cantidad de nitrógeno será igual a:

$$
13 \times 0.04=0.52 \% \text { de nitrógeno. }
$$

Puede emplearse también, el método de, MYERs, modificado por Gradwohl y Blaivas y cuya técnica es la siguiente:

Tómese un centímetro cúbico de orina y dilúyase en 25 c.c. de agua destilada. De la dilución así obtenida, se toma un centímetro cúbico en un tubo de prueba, cuidando de añadirle unas cinco a siete gotas de ácido sulfúrico concentrado, unos cinco a diez centígramos de sulfato de potasio y una gota de una solución de sulfato de cobre al $10 \%$. Se calienta el tubo, agitándolo continuamentè, hasta que el contenido tome una coloracion bruno oscura. Enfríese ligeramente y agrégese al líquido una gota de agua oxigenada: si no se aclara el contenido, se le lleva a la ebullición durante

(1) Él procedimiento empleado para el dosaje del aitrógeno total en la orina as el de KJeldah. 
un minuto, hasta que se obtenga un líquido claro. Solo entonces se deja enfríar el tubo por un minuto y luego, en un frasco graduado, se añade agua destilada hasta completar 35 centímetros cúbicos. Así queda preparado el frasco que llamaremos frasco A, para mayor claridad de la explicación.

El segundo frasco, que llamaremos $B$, está ocupado por la dilución, de 5 centímetros cúbicos de sulfato de amonio (que contiene un milígramo de Nitrogeno) en 30 c.c. cúbicos de agua destilada.

Al contenido de este segundo frasco se mezclan 10 centímetros cúbicos de una solución de Nessler en 40 centímetros cúbicos de agua destilada, hasta completar 50 centímetros cúbicos.

En el frasco A, que contiene la orina, se agrega el reactivo de Nessler diluído como en el frasco B: agrégase lentamente, moviendo el frasco receptor, con un movimiento giratorio, hasta que el álcali de NESSLER haya neutralizado el ácido sulfúrico del frasco A.

Se compara cuidadosamente al colorímetro, las coloraciones obtenidas y se hace el cálculo correspondientes a las diluciones. En esta forma se obtiene la cantidad de nitrógeno en $\frac{1}{25}$ de centímetro cúbico de orina, cifra que permite calcular fácilmente el porcentaje correspondiente. (6)

Azoemia nictemera.-Esta interesante investigación clínica comprende las siguientes operaciones:

1)-Dosaje de la urea en la orina eliminada en la mañana, antes del desayuno, habiendo en cuenta la hora de la miccion precedente, en el curso de la noche.

2)-Dosaje de la urea en la orina eliminada una hora despues de iniciado el período digestivo o sea dos horas después del almuerzo, que se toma de preferencia a la comida, para facilitar la investigacion.

Al estado normal los dosajes así practicados acusan: una disminución considerable de urea en la sangre, en el primer dosaje y un aumento máximo en el segundo la relación normal es de 20 a 100.

(6) R. B. H. GRad forl and A. Y. BLAivas.-A simple method for the determl nation of nitrogen in urine.-The Journal of the Amertcan Medical Assoctation. Vol. LXVII. No. 11. pág. 809 september 9. 1916. 
64

Anales

Indicaciones útiles. - Debo considerar entre los ureo metros utilizados en la apreciación volumétrica de la urea: los aparatos de Moreigne, Regnard, ivon, Doremus, Esbach, Auguy, Garcia y Bouriez, cuya descripción se halla en los tratados de Química Biologica: el urebmetro del Dr. Garcia está descrito en la sMemoria de la Municipalidad de Lima" (Año de 1906).

Es posible que alguno de estos urebmetros no venga acompanado de lás tablas necesarias para la estimación de la urea en la orina. En semejante caso, debe recurirse a la llamada iformula de correccion del volumen de nitrógeno medido en el urebmetron, que es la siguiente:

$$
V^{0}=V\left(\frac{1}{1 \times 0.00366 \times t}\right) \times \frac{H-f}{760}
$$

Los valores indicados en la formula son los siguientes:

$\mathrm{Vo}^{\mathrm{O}}=$ volúmen corregido a $0^{\circ}$ y $760 \mathrm{~m} . \mathrm{m}$.

$\mathrm{V}=$ volúmen leído en el ureómetro

$\mathrm{H}=$ presion atmosférica

$\mathrm{t}=$ temperatura

$\mathrm{f}=$ fuerza elástica del vapor.

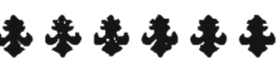

